\title{
Experiments on Neutron Transport through Concrete Member and the Potential for the Use in Material Investigation
}

\author{
T. Piotrowski ${ }^{a, *}$, D.B. Tefelski ${ }^{b}$, J. SkUbalski $^{c}$ AND A. ŻAK ${ }^{d}$ \\ ${ }^{a}$ Warsaw University of Technology, Faculty of Civil Engineering, Warsaw, Poland \\ ${ }^{b}$ Warsaw University of Technology, Faculty of Physics, Warsaw, Poland \\ ${ }^{c}$ Medical University, Department of Synthesis and Technology of Drugs, Łódź, Poland \\ ${ }^{d}$ University of Łódź, Faculty of Physics and Applied Informatics, Łódź, Poland
}

\begin{abstract}
Concrete has been used as a shield against high-energy photons and neutrons since the beginning of use of nuclear reaction in energy, medicine and research. From that time the progress in concrete technology is huge - very good concrete used in 60's was about $30 \mathrm{MPa}$ compressive strength, and now the recommendation of ETC-C is to use in EPR nuclear power plant the concrete at least class C45/55 for airplane resistant shell or class C40/50 for other structures. The Monte Carlo computer simulations indicate that an increase in density of the shielding member has a minor effect on the weakening of neutron transport and, therefore, the optimal composition of a shielding concrete against gamma radiation is different than the optimal composition of shielding concrete against neutron radiation. Neutron stopping is a two-step effect: slowing down of fast neutrons and absorption of thermal ones. Both result from the atomic composition of the barrier. The paper presents an analysis of neutrons transport through concrete cement mortar and polymer cement composites mortar based on specially designed experiments which allows for measuring fast neutron attenuation and thermal neutron capture separately. The aim of experiments was to find an influence of the cement type, polymer addition and moisture content on both aspects of neutron shielding properties of a composite. The experimental results were confirmed in MCNP simulations. There was found an influence of cement type on fast neutron attenuation due to differences in chemical composition. Next an important improvement of both fast neutron attenuation and thermal neutron capture due to polymer modification of concrete. The last was fond of clear linear relationship between fast neutron attenuation to the hydrogen content which shows the possibility of using this phenomenon in building material investigation like measurement of moisture content or polymer content in the polymer cement composites.
\end{abstract}

DOI: 10.12693/APhysPolA.128.B-14

PACS: 28.20.Fc, 28.41.Qb

\section{Introduction}

Concrete is one of the basic material using as a shielding against ionizing radiation because it is not very expensive and takes a construction role as well. Moreover as it is a composite, it is possible to optimize its composition for the best technical properties in given purpose. The main problem in optimization of concrete against ionizing radiation is a fact that while in case of protection against gamma the increase of density e.g. by the use of heavy-weight concrete, is usually sufficiently effective (but not so economic), for the protection against neutron this solution has a little effect on.

Akkurt and Elkhayat [1] found that the neutron attenuation coefficients decreased linearly with the increase of barite proportion in the concrete that is in opposition to photon attenuation coefficients. They showed that attenuation coefficients of neutron and gamma-rays are linearly increased and decreased, respectively, with increasing the proportion of hydrogen and moderators in concretes. It is due to the fact that the interaction of the neutrons with the concrete member depends both on the energy of neutrons and the probability for the given

${ }^{*}$ corresponding author; e-mail: t.piotrowski@il.pw.edu.pl reactions of neutrons and nuclei of atoms in the concrete called cross-section. That is why the use of marble [2] does not give an improvement of shielding properties of concrete against neutron radiation as the marble rate change the neutron attenuation in a negative way. It was explained by the fact that the concept of removal crosssection is based upon the presence of hydrogen (and by lower degree on the presence of the other moderator materials, $\mathrm{A} \leq 16$, like $\mathrm{C}$ and $\mathrm{O}$ ) in the absorber. This discussion is justified in the light of the results obtained by Malkapur et al. [3] from the study on the effect of hydrogen loading on the neutron radiation shielding characteristics of latex modified concrete mixes. An increasing trend of total neutron cross-section and decreasing trend of dose transmission values with increased hydrogen content was observed in comparison with control concrete with a few exceptions.

This concept has been partially rejected by Kharita et al. [4] who found that linear attenuation coefficients in the field of neutrons from Am-Be source for the heavyweight hematite concrete is about 10\% higher than for concrete samples expected to be suitable for neutron shielding that was a concrete with high hydrogen content prepared with serpentine aggregate. Similar results were presented by Gallego et al. [5] who analysed magnetite-based concrete and found its advantageous behaviour, when comparing neutron attenuation 
from ${ }^{241} \mathrm{Am}$-Be source against the thickness of the shielding with regard to ordinary concrete. Different are the results of studies made by Tefelski et al. [6] who compared neutron shielding calculated using MCNP code for ordinary, heavy-weight barite and reinforced concrete and concluded that the worst concrete for this purpose is barite heavy-weight concrete as it is full of high elements with very small cross-sections for neutron capture. In the same research it was proved that neutron attenuation for borated concrete in comparison to heavy concrete is of one or two orders of magnitude, but this advantage of borated concretes is removed as they have been the most activated and their activation decrease in time has been the slowest. On that background Akkurt and Elkhayat [1] stated that the most effective shielding material for mixed neutron and gamma-rays is obtained by mixed hydrogenous materials, heavy metal elements, and other neutron absorbers. In Poland, from the 60's it is assumed that the concrete containing 8-10\% water (free and chemically bound) with the possible addition of boron silicate (about $70 \mathrm{~kg} / \mathrm{m}^{3}$ ) and thickness necessary to shield the gamma radiation from reactor will also be effective enough for neutron radiation [8].

First studies carried out by Piotrowski et al. [9] in this field using Monte Carlo computer simulations indicate that both the compressive strength and type of concrete have a significant effect on the shielding against neutron. The decrease in the dose is proportional to the strength and an increase in the cement content and the associated mass of bound water as a result of its hydration. It was found, however, that the type of cement did not significantly affect the shielding properties. There was presented an advantage of the heavy-weight barite concrete over a normal-weight concrete in radiation beam corresponding to the reactor, but there was observed some disturbance in the thickness of approximately $25 \mathrm{~cm}$. The results of the MCNP simulations for mono-energetic neutrons with energies from $1 \mathrm{MeV}$ to $10 \mathrm{MeV}$ has shown that heavy-weight barite concrete was inferior to the weakening of radiation up to a thickness of $30-40 \mathrm{~cm}$, when a change in this relation was found.

Due to inconsistency of the results that has been already presented in the papers the aim of this research is to analyze the fast neutron attenuation and thermal neutron capture separately as they are the basic phenomena in neutron shielding (described below) but they depend on the different characteristics of concrete.

\section{Neutron transport through a concrete member}

The collisions of neutrons with the nuclei of atoms of the member have the major importance in the process of neutron transport through concrete. As they occur relatively rarely, in comparison to other radiation, neutrons (especially these of high energy - fast ones) can penetrate very thick layers of materials [4]. Depending on the kinetic energy of neutrons (speed) and the type on nuclei there may occur different phenomena: scattering (elastic and inelastic), absorption (capture radiation $(\mathrm{n}, \gamma)$ or with charged particles emitted), the nuclear reaction $(\mathrm{n}, 2 \mathrm{n})$ or $(\mathrm{n}, 3 \mathrm{n})$ and the fission reaction. From the neutron flux reduction point of view the most important are the first two. Scattering occurs when there is an exchange of kinetic energy between the neutron and the nuclei. There is possible an elastic scattering, in which, as in mechanics, energy is lost by the neutron proportionally to the ratio of the mass of the neutron and the mass of the nucleus which it has collided with. Inelastic scattering occurs when the neutron energy is greater than the energy of the lowest excited state of the nuclei (typically $>1 \mathrm{MeV}$ ). Then the neutron is "captured" by the nuclei, and the composed excited nuclei disintegrates with the emission of a neutron of a different energy. As a result of absorption a second important phenomenon is a nuclear reaction involving the capture of a neutron by the nucleus.

A highly excited intermediate nucleus has an excess of energy and emits it through nuclear transformation, which is accompanied by the emission of gamma rays or nuclear fission. A measure of the probability of occurrence of the given reaction of neutrons with the nucleus are named "cross-sections", defined as the effective area in which a neutron must hit to produce a specific phenomenon. As the cross-section for absorption of neutrons of high energies is small, in order to get the neutron flux weakening in the concrete shielding, it should be optimized so that the atoms of which it is composed: first caused precipitation of energy in the processes of elastic and inelastic scattering, and then absorption of slow neutrons. Protection against neutron radiation is therefore a two-step process $(1$ - slowing down, 2 - absorption). Slowing down of fast neutrons is mainly due to elastic collisions with the nuclei of light elements (like hydrogen) and inelastic scattering on nuclei of heavy elements, for which the energy of the lowest excited state is much less than for light elements (e.g. $\mathrm{Pb}-0.4 \mathrm{MeV}, \mathrm{O}-$ 6.0 MeV). The exact calculation is possible by numerical calculations, but till now there is no reliable method of calculation, which could be recommended by building engineers for practical use.

\section{Experiments}

In order to meet the aim of the research two experimental programs were performed. The first was measurement of the degree of absorption of thermal neutrons in the concrete of a certain thickness and the other was measurement of the degree of slowing down fast neutrons to thermal neutrons. Interpretation of the results means, the better concrete is, more neutrons in the first study absorbs and at the same time the larger number of fast neutrons slows down in the second study (it is measured indirectly by an increase in the number of registered thermal neutrons). In both cases the helium counters were used and neutrons were emitted by a $\mathrm{Pu}-\mathrm{Be}$ source. To eliminate fast neutrons in the first study the neutrons were 
attenuated to thermal ones by elastic scattering and inelastic collisions of fast neutrons with hydrogen atoms which are part of the moderator - paraffin surrounding the source in a special paraffin block.

Schematic representation of the measuring system for the first experimental program in a block of paraffin is shown in Fig. 1.

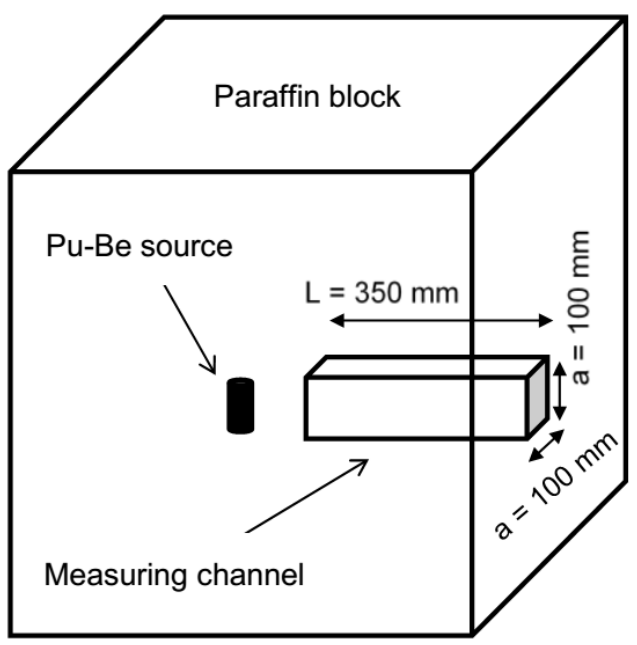

Fig. 1. Paraffin block used in thermal neutron absorption measurement.

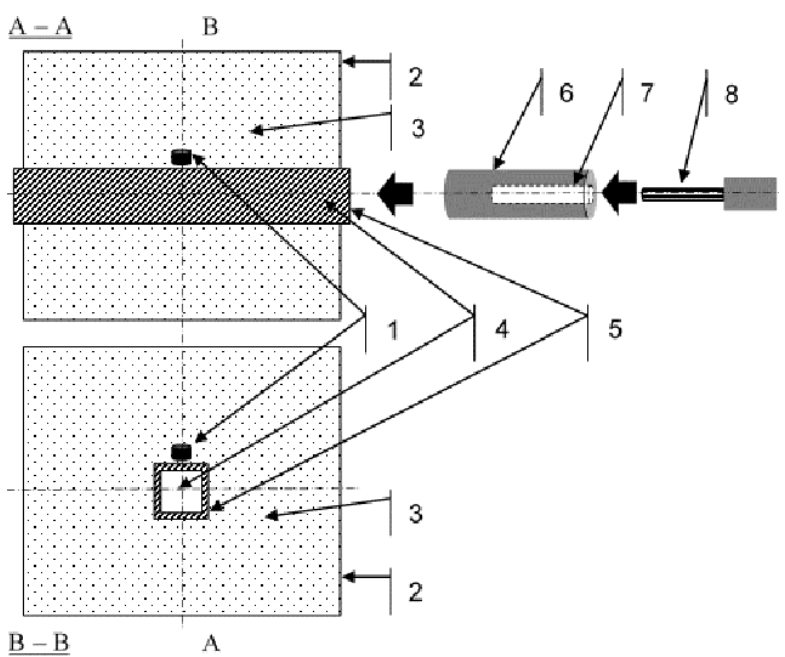

Fig. 2. The system for fast neutron attenuation measurement with cadmium sheet channel: $1-\mathrm{Pu}-\mathrm{Be}$ neutron source; 2 - outside barrier; 3 - paraffin block; 4 - measuring channel; 5 - metal cadmium sheet; 6 studied sample; 7 - measuring hole for helium counter; 8 - helium counter with preamplifier.

In the second experiment the other system was built in order to stop all the thermal neutrons (Fig. 2) and allow only fast neutrons to penetrate the sample. It is made by using a filter sheet made of cadmium, which absorbs thermal neutrons very well [10]. As only fast neutrons emitted by the source pass into the measuring channel, in which the concrete sample is, a helium counter placed inside the sample records only thermal neutrons that are a result of slowing fast neutrons in a sample of concrete. This should be mainly an effect of collisions with the hydrogen atoms contained in the sample. This relation could be proved by drawing the correlation of the number of neutrons recorded and the degree of hydration (moisture content) of concrete. For the experiments specific samples were prepared, allowing to place a detector inside the cylindrical sample in a special hole (Fig. 3).
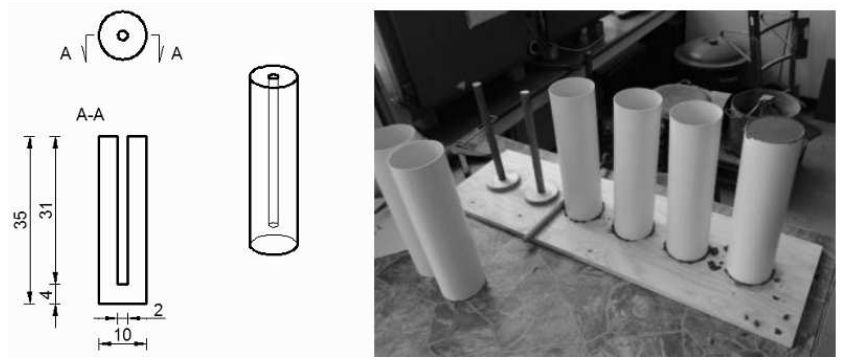

Fig. 3. Samples used in experiments.

To determine the effect of cement on the shielding properties the test was made on standard cement mortar CEM I $42.5 \mathrm{R}, \mathrm{CEM}$ III/A $42.5 \mathrm{~N}-\mathrm{LH} / \mathrm{HSR} / \mathrm{NA}$ and $\mathrm{CEM} \mathrm{IV/B}(\mathrm{V}) 32.5-\mathrm{LH} / \mathrm{HSR}$, which differs in the relative content of the dominant elements including $\mathrm{Al}$, $\mathrm{Fe}$, and $\mathrm{Ca}$. To determine the effect of polymer addition, the studies have also been carried out for three polymer cement mortars (polymer cement composites PCC): standard cement mortar with the addition of the $10 \%$ polymer dispersion in relation to cement content PCC $(\mathrm{ZN}+\mathrm{P})$ and two commercially available concrete repair mortar according to EN 1504-3 marked as PCC-1 and PCC-2.

\section{Results}

The results are presented in relative number of registered thermal neutrons $(W c p)$ by the helium counter in relation to a fixed initial value - background $\left(c p s_{0}\right)$ according to

$$
W c p=\frac{\Delta\left[c p s_{0} ; c p s_{t}\right]}{c p s_{0}} 100 \%,
$$

where $W c p$ - the relative number of registered thermal neutrons, $c p s_{t}$ - the number of registered thermal neutrons emerging from a sample of concrete, $c p s_{0}$ — the number of registered thermal neutrons without concrete samples in the test channel.

In relation to the initial number of thermal neutrons in the paraffin block, the $4 \mathrm{~cm}$ thick cement mortar retains $62-63 \%$, regardless of the type of cement used (Fig. 4). Addition of polymer proved to be advantageous - absorption ranges from 64 to $69 \%$ and is directly proportional to the compressive strength. Analyzing attenuation of fast neutrons there can be observed a significant influence of the type of cement. Standard cement mortar 
with Portland cement CEM I has almost twice greater slowing of fast neutron flux $(46 \%)$ than the mortar of other cements $(26-27 \%)$. The impact of the polymer to slow down neutrons is clear as well - PCC mortars show slowing up to twice more effective than cement mortars.

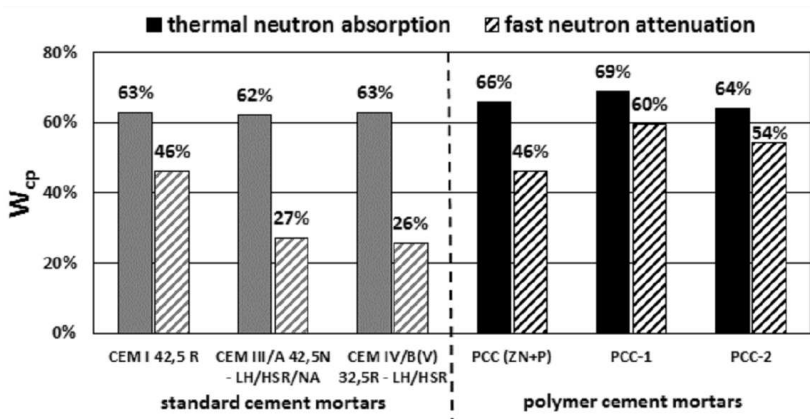

Fig. 4. The results of the thermal neutron absorption and slowing down fast neutrons.

Slowing down fast neutrons measurement was also performed for samples of different moisture content and resulting hydrogen content. One can observe a clear linear relationship between the fast neutron attenuation to the moisture content (Fig. 5). The results of the relative weakening of fast neutron flux and the slope of the trend line for the PCC mortars are slightly higher than for standard cement mortars. It can be assumed that each $\%$ of moisture content makes a $15 \%$ increase of fast neutron attenuation.

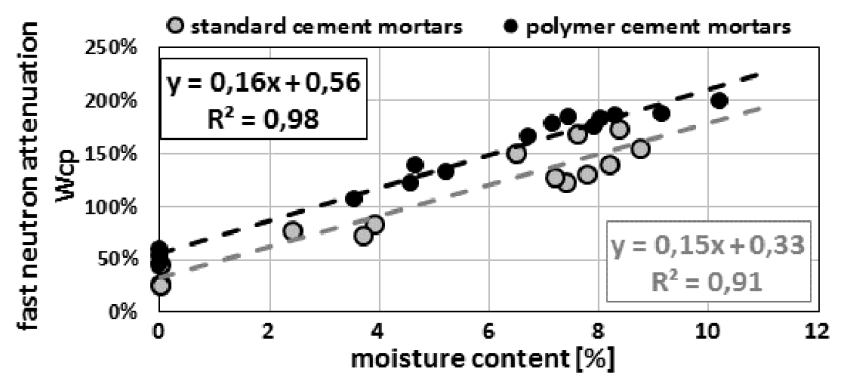

Fig. 5. Results of slowing down fast neutrons to thermal neutrons in samples of standard cement mortars and PCC mortars depending on the moisture content.

The experimental results were in accordance to MCNP simulations made for the same geometry and conditions $(\mathrm{Pu}-\mathrm{Be}$ source, helium neutron detector) as it was in the first experiment. The details of performed MCNP simulations are described in a study of Piotrowski et al. [11]. It is proved by close values of linear attenuation coefficient - $\mu$, half value layer - HVL and tenth value layer - TVL values (Table) calculated as follows [12, 13]:

$$
\mu=\frac{1}{x} \ln \frac{I_{0}}{I}\left[\mathrm{~cm}^{-1}\right], \quad \mathrm{HVL}=\frac{\ln 2}{\mu} ;
$$

$$
\mathrm{TVL}=\frac{\ln 10}{\mu},
$$

where $x$ is the material thickness and $I$ and $I_{0}$ are the background subtracted number of counts recorded in detector with and without concrete material between detector and source, respectively.

TABLE

Experimental and MCNP simulated values of linear attenuation coefficient, HVL and TVL.

\begin{tabular}{l|c|c}
\hline \hline Parameter & Experimental & MCNP \\
\hline $\begin{array}{l}\text { Neutrons on the detector from } \\
\text { the source (no sample) }\end{array}$ & $100 \%$ & $100 \%$ \\
\hline $\begin{array}{l}\text { neutrons on the detector } \\
\text { behind the sample }\end{array}$ & $36.6 \%$ & $36.1 \%$ \\
\hline $\begin{array}{l}\text { linear attenuation coefficient, } \\
\mu\left[\mathrm{cm}^{-1}\right]\end{array}$ & 0.251 & 0.249 \\
\hline half value layer, HVL $[\mathrm{cm}]$ & 2.76 & 2.78 \\
\hline tenth value layer, TVL $[\mathrm{cm}]$ & 9.16 & 9.25
\end{tabular}

\section{Conclusion}

The results of designed experiment are in accordance to MCNP calculations. It was proved that neutron shielding is related to two phenomena - fast neutron attenuation and thermal neutron absorption, and they are both depending on the atomic compositions of concrete but not in the same way. The designed experimental setup allows for an analysis of both aspects separately. For example, fast neutron attenuation is influenced by the cement type and its atomic composition but these differences are not present in thermal neutron absorption. Additionally, it was confirmed a significant benefit on neutron shielding properties obtained by polymer addition. PCC repair mortars exhibit superior properties in terms of both thermal neutron absorption and fast neutrons attenuation. This is probably due to bigger number of the hydrogen atoms resulting from the content of the hydrocarbon chains of the polymers. Linear correlation in the measurements of slowing down fast neutrons with different moisture content confirms the influence of hydrogen content on neutron thermalization. Due to that, there is a possibility of using this type of measurement methods in material test like measurement of moisture content or polymer content in the PCC.

\section{Acknowledgments}

This article is part of Project No. LIDER/033/639/L4/12/NCBIR/2013 entitled NGS-Concrete New Generation concrete shielding against ionizing radiation, in the framework of the IVth edition of the Leader Program implemented by NCBiR and was additionally supported by the Faculty of Civil Engineering of Warsaw University of Technology in 2014. Thanks to Lafarge Cement SA in Poland for providing the cement and Surico MT Surosz Sp. J. for magnetite aggregate for research. 


\section{References}

[1] I. Akkurt, A. Elkhayat, Ann. Nucl. Energy 51, 5 (2013).

[2] A. Elkhayat, I. Akkurt, Ann. Nucl. Energy 60, 8 (2013)

[3] S.M. Malkapur, H. Satdive, M.C. Narasimhan, N.B. Karkera, P. Goverdhan, V. Sathian, Prog. Nucl. Energy 83, 8 (2015).

[4] M.H. Kharita, M. Takeyeddin, M. Alnassar, S. Yousef, Prog. Nucl. Energy 50, 33 (2008).

[5] E. Gallego, A. Lorente, H.R. Vega-Carrillo, Nucl. Technol. 168, 399 (2009).

[6] D.B. Tefelski, T. Piotrowski, V. Bildeanu, A. Polanski, J. Skubalski, Bull. Pol. Acad. Sci. Tech. Sci. 61, 161 (2013).

[7] www.nrc.gov (United States Nuclear Regulatory Commission web page).
[8] E. Pohl, Nuclear Technics in Civil Engineering, Arkady, Warsaw 1967 (in Polish).

[9] T. Piotrowski, D.B. Tefelski, A. Polański, J. Skubalski, Open Eng. 2, 296 (2012).

[10] I. Sirakov, S. Kopecky, C. Massimi, M. Moxon, V. Pronyaev, P. Schillebeeckx, A. Trkov, K. Volev, R. Wynants, ENDF-6 compatible evaluation of neutron induced reaction cross sections for ${ }_{106,108,110,111,112,113,114,116}$ Cd, EUR 25800 - Joint Research Centre - Institute for Reference Materials and Measurements, European Commission, Luxembourg 2013.

[11] T. Piotrowski, D.B. Tefelski, J.J. Sokołowska, B. Jaworska, Acta Phys. Pol. A 128, A-9 (2015).

[12] I. Akkurt, R. Altindag, K. Gunoglu, H.R. Sarıkaya, Ann. Nucl. Energy 43, 56 (2012).

[13] I. Akkurt, H. Akyildirim, Nucl. Eng. Des. 252, 163 (2012). 